\title{
Existence and Uniqueness Results for Hadamard-Type Fractional Differential Equations with Nonlocal Fractional Integral Boundary Conditions
}

\author{
Phollakrit Thiramanus, ${ }^{1}$ Sotiris K. Ntouyas, ${ }^{2}$ and Jessada Tariboon ${ }^{1}$ \\ ${ }^{1}$ Nonlinear Dynamic Analysis Research Center, Department of Mathematics, Faculty of Applied Science, \\ King Mongkut's University of Technology North Bangkok, Bangkok 10800, Thailand \\ ${ }^{2}$ Department of Mathematics, University of Ioannina, 45110 Ioannina, Greece
}

Correspondence should be addressed to Jessada Tariboon; jessadat@kmutnb.ac.th

Received 1 April 2014; Accepted 10 June 2014; Published 26 June 2014

Academic Editor: Bashir Ahmad

Copyright (C) 2014 Phollakrit Thiramanus et al. This is an open access article distributed under the Creative Commons Attribution License, which permits unrestricted use, distribution, and reproduction in any medium, provided the original work is properly cited.

We study the existence and uniqueness of solutions for a fractional boundary value problem involving Hadamard-type fractional differential equations and nonlocal fractional integral boundary conditions. Our results are based on some classical fixed point theorems. Some illustrative examples are also included.

\section{Introduction}

In this paper, we investigate the following Hadamard boundary value problem:

$$
\begin{gathered}
D^{q} x(t)=f(t, x(t)), \quad 1<q \leq 2, t \in(1, e), \\
x(1)=0, \quad \sum_{i=1}^{m} \lambda_{i} J^{\alpha_{i}} x\left(\eta_{i}\right)=\sum_{j=1}^{n} \mu_{j}\left(J^{\beta_{j}} x(e)-J^{\beta_{j}} x\left(\xi_{j}\right)\right),
\end{gathered}
$$

where $D^{q}$ denotes the Hadamard fractional derivative of order $q, f:[1, e] \times \mathbb{R} \rightarrow \mathbb{R}$ is a continuous function, $\eta_{i}, \xi_{j} \in(1, e), \lambda_{i}, \mu_{j} \in \mathbb{R}$, for all $i=1,2, \ldots, m, j=1,2, \ldots, n$, $\eta_{1}<\eta_{2}<\cdots<\eta_{m}, \xi_{1}<\xi_{2}<\cdots<\xi_{n}$, and $J^{\phi}$ is the Hadamard fractional integral of order $\phi>0\left(\phi=\alpha_{i}, \beta_{j}, i=\right.$ $1,2, \ldots, m, j=1,2, \ldots, n)$.

We mention that integral boundary conditions are encountered in various applications such as population dynamics, blood flow models, chemical engineering, cellular systems, heat transmission, plasma physics, and thermoelasticity.

Condition (2) is a general form of the integral boundary conditions considered in [1] and covers many special cases.
For example, if $\alpha_{i}=\beta_{j}=1$, for all $i=1,2, \ldots, m$ and $j=1,2$, $\ldots, n$, then condition (2) reduces to

$$
\begin{gathered}
x(1)=0 \\
\lambda_{1} \int_{1}^{\eta_{1}} x(s) \frac{d s}{s}+\cdots+\lambda_{m} \int_{1}^{\eta_{m}} x(s) \frac{d s}{s} \\
=\mu_{1} \int_{\xi_{1}}^{e} x(s) \frac{d s}{s}+\cdots+\mu_{n} \int_{\xi_{n}}^{e} x(s) \frac{d s}{s} .
\end{gathered}
$$

Fractional differential equations provide appropriate models for describing real world problems, which cannot be described using classical integer order differential equations. The theory of fractional differential equations has received much attention over the past years and has become an important field of investigation due to its extensive applications in numerous branches of physics, economics, and engineering sciences [2-5]. Some recent contributions to the subject can be seen in $[1,6-20]$ and references cited therein.

It has been noticed that most of the work on this topic is based on Riemann-Liouville and Caputo type fractional differential equations. Another kind of fractional derivatives that appears side by side to Riemann-Liouville and Caputo derivatives in the literature is the fractional derivative due 
to Hadamard introduced in 1892 [21], which differs from the preceding ones in the sense that the kernel of the integral (in the definition of Hadamard derivative) contains logarithmic function of arbitrary exponent. Details and properties of Hadamard fractional derivative and integral can be found in $[2,22-26]$. For some recent results on Hadamard boundary value problem we refer to $[27,28]$.

We establish a variety of results for the problem (1)(2) by using classical fixed point theorems. The first result, Theorem 4, relies on Banach contraction mapping principle and concerns an existence and uniqueness result for the solutions of the problem (1)-(2). A second existence and uniqueness result is proved in Theorem 7, via nonlinear contractions and a fixed point theorem due to Boyd and Wong. Existence results are proved in the third result, Theorem 9, by using Krasnoselskii fixed point theorem, and in the fourth result, Theorem 12, by using nonlinear alternative of LeraySchauder type.

The paper is organized as follows. In Section 2, we recall some preliminary concepts that we need in the sequel and prove a preliminary lemma. Section 3 contains the main results for the problem (1)-(2). In Section 4, some illustrative examples are discussed.

\section{Preliminaries}

In this section, we introduce some notations and definitions of fractional calculus [2] and present preliminary results needed in our proofs later.

Definition 1. The Hadamard derivative of fractional order $q$ for a function $f:[1, \infty) \rightarrow \mathbb{R}$ is defined as

$$
\begin{array}{r}
D^{q} f(t)=\frac{1}{\Gamma(n-q)}\left(t \frac{d}{d t}\right)^{n} \int_{1}^{t}\left(\log \frac{t}{s}\right)^{n-q-1} \frac{f(s)}{s} d s, \\
n-1<q<n, n=[q]+1,
\end{array}
$$

where $[q]$ denotes the integer part of the real number $q$, $\log (\cdot)=\log _{e}(\cdot)$, and $\Gamma$ is the Gamma function.

Definition 2. The Hadamard fractional integral of order $q$ for a function $f:[1, \infty) \rightarrow \mathbb{R}$ is defined by

$$
J^{q} f(t)=\frac{1}{\Gamma(q)} \int_{1}^{t}\left(\log \frac{t}{s}\right)^{q-1} \frac{f(s)}{s} d s, \quad q>0,
$$

provided the integral exists.

For convenience, we set

$$
\begin{aligned}
\Lambda= & \sum_{i=1}^{m} \lambda_{i} \frac{\Gamma(q)}{\Gamma\left(q+\alpha_{i}\right)}\left(\log \eta_{i}\right)^{q+\alpha_{i}-1} \\
& -\sum_{j=1}^{n} \mu_{j} \frac{\Gamma(q)}{\Gamma\left(q+\beta_{j}\right)}\left(1-\left(\log \xi_{j}\right)^{q+\beta_{j}-1}\right) .
\end{aligned}
$$

Lemma 3. Let $\Lambda \neq 0,1<q \leq 2, \alpha_{i}, \beta_{j}>0$, and $\eta_{i}, \xi_{j} \in(1, e)$ for $i=1,2, \ldots, m, j=1,2, \ldots, n$, and $h \in C([1, e], \mathbb{R})$. The unique solution of the following fractional differential equation,

$$
D^{q} x(t)=h(t), \quad t \in(1, e),
$$

subject to the boundary condition,

$$
x(1)=0, \quad \sum_{i=1}^{m} \lambda_{i} J^{\alpha_{i}} x\left(\eta_{i}\right)=\sum_{j=1}^{n} \mu_{j}\left(J^{\beta_{j}} x(e)-J^{\beta_{j}} x\left(\xi_{j}\right)\right),
$$

is given by the integral equation

$$
\begin{aligned}
x(t)= & \frac{(\log t)^{q-1}}{\Lambda} \sum_{j=1}^{n} \mu_{j}\left(J^{q+\beta_{j}} h(e)-J^{q+\beta_{j}} h\left(\xi_{j}\right)\right) \\
& -\frac{(\log t)^{q-1}}{\Lambda} \sum_{i=1}^{m} \lambda_{i} J^{q+\alpha_{i}} h\left(\eta_{i}\right)+J^{q} h(t) .
\end{aligned}
$$

Proof. Applying the Hadamard fractional integral of order $q$ to both sides of (7), we have

$$
x(t)=z_{1}(\log t)^{q-1}+z_{2}(\log t)^{q-2}+J^{q} h(t),
$$

where $z_{1}, z_{2} \in \mathbb{R}$.

The condition of $x(1)=0$ implies $z_{2}=0$. Therefore,

$$
x(t)=z_{1}(\log t)^{q-1}+J^{q} h(t) .
$$

For any $p>0$, by Definition 2, it follows that

$$
J^{p} x(t)=z_{1} \frac{\Gamma(q)}{\Gamma(q+p)}(\log t)^{q+p-1}+J^{q+p} h(t) .
$$

The second condition of (8) with (12) leads to

$$
z_{1}=\frac{1}{\Lambda} \sum_{j=1}^{n} \mu_{j}\left(J^{q+\beta_{j}} h(e)-J^{q+\beta_{j}} h\left(\xi_{j}\right)\right)-\frac{1}{\Lambda} \sum_{i=1}^{m} \lambda_{i} J^{q+\alpha_{i}} h\left(\eta_{i}\right) .
$$

Substituting the value of a constant $z_{1}$ into (11), we obtain (9) as required. The proof is completed.

\section{Main Results}

Let $\mathscr{C}=C([1, e], \mathbb{R})$ denote the Banach space of all continuous functions from $[1, e]$ to $\mathbb{R}$ endowed with the norm defined by $\|x\|=\sup _{t \in[1, e]}|x(t)|$. As in Lemma 3, we define an operator $\mathscr{F}: \mathscr{C} \rightarrow \mathscr{C}$ by

$$
\begin{aligned}
(\mathscr{F} x)(t)= & J^{q} f(s, x(s))(t) \\
& -\frac{(\log t)^{q-1}}{\Lambda} \sum_{i=1}^{m} \lambda_{i} J^{\alpha_{i}+q} f(s, x(s))\left(\eta_{i}\right) \\
& +\frac{(\log t)^{q-1}}{\Lambda} \sum_{j=1}^{n} \mu_{j}\left(J^{\beta_{j}+q} f(s, x(s))(e)\right. \\
& \left.\quad-J^{\beta_{j}+q} f(s, x(s))\left(\xi_{j}\right)\right),
\end{aligned}
$$

with $\Lambda \neq 0$. It should be noticed that problem (1)-(2) has solutions if and only if the operator $\mathscr{F}$ has fixed points. 
For the sake of convenience, we put

$$
\begin{aligned}
\Phi= & \frac{1}{\Gamma(q+1)}+\frac{1}{|\Lambda|} \sum_{i=1}^{m}\left|\lambda_{i}\right| \frac{\left(\log \eta_{i}\right)^{\alpha_{i}+q}}{\Gamma\left(\alpha_{i}+q+1\right)} \\
& +\frac{1}{|\Lambda|} \sum_{j=1}^{n}\left|\mu_{j}\right| \frac{1+\left(\log \xi_{j}\right)^{\beta_{j}+q}}{\Gamma\left(\beta_{j}+q+1\right)} .
\end{aligned}
$$

The first existence and uniqueness result is based on the Banach contraction mapping principle.

Theorem 4. Let $f:[1, e] \times \mathbb{R} \rightarrow \mathbb{R}$ be a continuous function satisfying the assumption that

$\left(\mathrm{H}_{1}\right)$ there exists a constant $L>0$ such that $\mid f(t, x)-$ $f(t, y)|\leq L| x-y \mid$, for each $t \in[1, e]$ and $x, y \in \mathbb{R}$.

If

$$
L \Phi<1
$$

where $\Phi$ is given by (15), then the boundary value problem (1)(2) has a unique solution on $[1, e]$.

Proof. We transform the problem (1)-(2) into a fixed point problem, $x=\mathscr{F} x$, where the operator $\mathscr{F}$ is defined by (14). By using Banach's contraction mapping principle, we will show that $\mathscr{F}$ has a fixed point which is a unique solution of problem (1)-(2).

We set $\sup _{t \in[1, e]}|f(t, 0)|=M<\infty$ and choose

$$
r \geq \frac{M \Phi}{1-L \Phi}
$$

Now, we show that $\mathscr{F} B_{r} \subset B_{r}$, where $B_{r}=\{x \in \mathscr{C}:\|x\| \leq$ $r$. For any $x \in B_{r}$, we have

$$
\begin{aligned}
& \|\mathscr{F} x\| \\
& \qquad \sup _{t \in[1, e]}\left\{J^{q}|f(s, x(s))|(t)\right. \\
& +\frac{(\log t)^{q-1}}{|\Lambda|} \sum_{i=1}^{m}\left|\lambda_{i}\right| J^{\alpha_{i}+q}|f(s, x(s))|\left(\eta_{i}\right) \\
& +\frac{(\log t)^{q-1}}{|\Lambda|} \sum_{j=1}^{n}\left|\mu_{j}\right|\left(J^{\beta_{j}+q}|f(s, x(s))|(e)\right. \\
& \left.\left.+J^{\beta_{j}+q}|f(s, x(s))|\left(\xi_{j}\right)\right)\right\}
\end{aligned}
$$

$$
\begin{aligned}
& \leq J^{q}(|f(s, x(s))-f(s, 0)|+|f(s, 0)|)(e) \\
& +\frac{1}{|\Lambda|} \sum_{i=1}^{m}\left|\lambda_{i}\right| J^{\alpha_{i}+q}(|f(s, x(s))-f(s, 0)|+|f(s, 0)|)\left(\eta_{i}\right) \\
& +\frac{1}{|\Lambda|} \sum_{j=1}^{n}\left|\mu_{j}\right|\left(J^{\beta_{j}+q}(|f(s, x(s))-f(s, 0)|+|f(s, 0)|)(e)\right. \\
& +J^{\beta_{j}+q}(|f(s, x(s))-f(s, 0)| \\
& +(\operatorname{Lr}+M)\left\{\frac{1}{\Gamma(q+1)}+\frac{1}{|\Lambda|} \sum_{i=1}^{m}\left|\lambda_{i}\right| \frac{\left(\log \eta_{i}\right)^{\alpha_{i}+q}}{\Gamma\left(\alpha_{i}+q+1\right)}\right. \\
& \left.+\frac{1}{|\Lambda|} \sum_{j=1}^{n}\left|\mu_{j}\right| \frac{1+\left(\log \xi_{j}\right)^{\beta_{j}+q}}{\Gamma\left(\beta_{j}+q+1\right)}\right\} \\
& =(\operatorname{Lr}+M) \Phi \leq r .
\end{aligned}
$$

It follows that $\mathscr{F} B_{r} \subset B_{r}$.

For $x, y \in \mathscr{C}$ and for each $t \in[1, e]$, we have

$$
\begin{aligned}
& |\mathscr{F} x(t)-\mathscr{F} y(t)| \\
& \leq J^{q}(|f(s, x(s))-f(s, y(s))|)(t) \\
& +\frac{(\log t)^{q-1}}{|\Lambda|} \sum_{i=1}^{m}\left|\lambda_{i}\right| J^{\alpha_{i}+q}(|f(s, x(s))-f(s, y(s))|)\left(\eta_{i}\right) \\
& +\frac{(\log t)^{q-1}}{|\Lambda|} \sum_{j=1}^{n}\left|\mu_{j}\right|\left(J^{\beta_{j}+q}(|f(s, x(s))-f(s, y(s))|)(e)\right. \\
& \quad+J^{\beta_{j}+q}(|f(s, x(s))-f(s, y(s))|) \\
& \leq L\|x-y\|\left\{\frac{1}{\Gamma(q+1)}+\frac{1}{|\Lambda|} \sum_{i=1}^{m}\left|\lambda_{i}\right| \frac{\left(\log \eta_{i}\right)^{\alpha_{i}+q}}{\Gamma\left(\alpha_{i}+q+1\right)}\right. \\
& \left.\times \frac{1}{|\Lambda|} \sum_{j=1}^{n}\left|\mu_{j}\right| \frac{1+\left(\log \xi_{j}\right)^{\beta_{j}+q}}{\Gamma\left(\beta_{j}+q+1\right)}\right\} \\
& =L \Phi\|x-y\| .
\end{aligned}
$$$$
+J^{\beta_{j}+q}(|f(s, x(s))-f(s, y(s))|)
$$

The above result implies that $\|\mathscr{F} x-\mathscr{F} y\| \leq L \Phi\|x-y\|$. As $L \Phi<1$, therefore $\mathscr{F}$ is a contraction. Hence, by the Banach contraction mapping principle, we deduce that $\mathscr{F}$ has a fixed point which is the unique solution of the problem (1)-(2).

Next, we give the second existence and uniqueness result by using nonlinear contractions.

Definition 5. Let $E$ be a Banach space and let $F: E \rightarrow E$ be a mapping. $F$ is said to be a nonlinear contraction if there exists 
a continuous nondecreasing function $\Psi: \mathbb{R}^{+} \rightarrow \mathbb{R}^{+}$such that $\Psi(0)=0$ and $\Psi(\theta)<\theta$ for all $\theta>0$ with the property

$$
\|F x-F y\| \leq \Psi(\|x-y\|), \quad \forall x, y \in E .
$$

Lemma 6 (see [29]). Let $E$ be a Banach space and let $F: E \rightarrow$ $E$ be a nonlinear contraction. Then $F$ has a unique fixed point in $E$.

Theorem 7. Let $f:[1, e] \times \mathbb{R} \rightarrow \mathbb{R}$ be a continuous function satisfying the assumption

$\left(\mathrm{H}_{2}\right)|f(t, x)-f(t, y)| \leq h(t)\left(|x-y| /\left(H^{*}+|x-y|\right)\right), t \in$ $[1, e], x, y \geq 0$, where $h:[1, e] \rightarrow \mathbb{R}^{+}$is continuous and a constant $H^{*}$ is defined by

$$
\begin{aligned}
H^{*}= & J^{q} h(e)+\frac{1}{|\Lambda|} \sum_{i=1}^{m}\left|\lambda_{i}\right| J^{\alpha_{i}+q} h\left(\eta_{i}\right) \\
& +\frac{1}{|\Lambda|} \sum_{j=1}^{n}\left|\mu_{j}\right|\left(J^{\beta_{j}+q} h(e)+J^{\beta_{j}+q} h\left(\xi_{j}\right)\right) .
\end{aligned}
$$

Then the boundary value problem (1)-(2) has a unique solution.

Proof. We define the operator $\mathscr{F}: \mathscr{C} \rightarrow \mathscr{C}$ as (14) and a continuous nondecreasing function $\Psi: \mathbb{R}^{+} \rightarrow \mathbb{R}^{+}$by

$$
\Psi(\theta)=\frac{H^{*} \theta}{H^{*}+\theta}, \quad \forall \theta \geq 0 .
$$

Note that the function $\Psi$ satisfies $\Psi(0)=0$ and $\Psi(\theta)<\theta$ for all $\theta>0$.

For any $x, y \in \mathscr{C}$ and for each $t \in[1, e]$, we have

$$
\begin{aligned}
& |\mathscr{F} x(t)-\mathscr{F} y(t)| \\
& \leq J^{q}(|f(s, x(s))-f(s, y(s))|)(t) \\
& +\frac{(\log t)^{q-1}}{|\Lambda|} \sum_{i=1}^{m}\left|\lambda_{i}\right| J^{\alpha_{i}+q}(|f(s, x(s))-f(s, y(s))|)\left(\eta_{i}\right) \\
& +\frac{(\log t)^{q-1}}{|\Lambda|} \sum_{j=1}^{n}\left|\mu_{j}\right|\left(J^{\beta_{j}+q}(|f(s, x(s))-f(s, y(s))|)(e)\right. \\
& \quad+J^{\beta_{j}+q}(|f(s, x(s))-f(s, y(s))|) \\
& \left.\quad \times\left(\xi_{j}\right)\right) \\
& +\frac{1}{|\Lambda|} \sum_{i=1}^{m}\left|\lambda_{i}\right| J^{\alpha_{i}+q}\left(h(s) \frac{|x(s)-y(s)|}{H^{*}+|x(s)-y(s)|}\right)\left(\eta_{i}\right) \\
& +\frac{1}{|\Lambda|} \sum_{j=1}^{n}\left|\mu_{j}\right|\left\{J^{\beta_{j}+q}\left(h(s) \frac{|x(s)-y(s)|}{H^{*}+|x(s)-y(s)|}\right)(e)\right. \\
& \left.+J^{\beta_{j}+q}\left(h(s) \frac{|x(s)-y(s)|}{H^{*}+|x(s)-y(s)|}\right)\left(\xi_{j}\right)\right\}
\end{aligned}
$$

$$
\begin{array}{r}
\leq \frac{\Psi(\|x-y\|)}{H^{*}}\left(J^{q} h(e)+\frac{1}{|\Lambda|} \sum_{i=1}^{m}\left|\lambda_{i}\right| J^{\alpha_{i}+q} h\left(\eta_{i}\right)\right. \\
\left.+\frac{1}{|\Lambda|} \sum_{j=1}^{n}\left|\mu_{j}\right|\left(J^{\beta_{j}+q} h(e)+J^{\beta_{j}+q} h\left(\xi_{j}\right)\right)\right)
\end{array}
$$$$
=\Psi(\|x-y\|) \text {. }
$$

This implies that $\|\mathscr{F} x-\mathscr{F} y\| \leq \Psi(\|x-y\|)$. Therefore $\mathscr{F}$ is a nonlinear contraction. Hence, by Lemma 6 the operator $\mathscr{F}$ has a fixed point which is the unique solution of the problem (1)-(2).

Next, we give an existence result by using Krasnoselskii's fixed point theorem.

Lemma 8 (Krasnoselskii's fixed point theorem [30]). Let $M$ be a closed, bounded, convex, and nonempty subset of a Banach space $X$. Let $A, B$ be the operators such that (a) $A x+B y \in M$, whenever $x, y \in M$; (b) $A$ is compact and continuous; (c) $B$ is a contraction mapping. Then there exists $z \in M$ such that $z=$ $A z+B z$.

Theorem 9. Assume that $f:[1, e] \times \mathbb{R} \rightarrow \mathbb{R}$ is a continuous function satisfying assumption $\left(H_{1}\right)$. In addition we suppose that

$$
\left(\mathrm{H}_{3}\right)|f(t, x)| \leq \kappa(t), \forall(t, x) \in[1, e] \times \mathbb{R} \text { and } \kappa \in
$$
$C\left([1, e], \mathbb{R}^{+}\right)$.

If

$$
\frac{L}{\Gamma(q+1)}<1
$$

then the boundary value problem (1)-(2) has at least one solution on $[1, e]$.

Proof. We define $\sup _{t \in[1, e]}|\kappa(t)|=\|\kappa\|$ and choose a suitable constant $\bar{r}$ as

$$
\bar{r} \geq\|\kappa\| \Phi,
$$

where $\Phi$ is defined by (15). Furthermore, we define the operators $\mathscr{P}$ and $\mathscr{Q}$ on $B_{\bar{r}}=\{x \in \mathscr{C}:\|x\| \leq \bar{r}\}$ as

$$
\begin{array}{r}
(\mathscr{P} x)(t)=\frac{(\log t)^{q-1}}{\Lambda} \sum_{j=1}^{n} \mu_{j}\left(J^{\beta_{j}+q} f(s, x(s))(e)\right. \\
\left.-J^{\beta_{j}+q} f(s, x(s))\left(\xi_{j}\right)\right) \\
-\frac{(\log t)^{q-1}}{\Lambda} \sum_{i=1}^{m} \lambda_{i} J^{\alpha_{i}+q} f(s, x(s))\left(\eta_{i}\right), \\
t \in[1, e],
\end{array}
$$


For $x, y \in B_{\bar{r}}$, we have

$$
\begin{aligned}
\|\mathscr{P} x+\mathscr{Q} y\| \leq\|\kappa\| & \frac{1}{\Gamma(q+1)}+\frac{1}{|\Lambda|} \sum_{i=1}^{m}\left|\lambda_{i}\right| \frac{\left(\log \eta_{i}\right)^{\alpha_{i}+q}}{\Gamma\left(\alpha_{i}+q+1\right)} \\
& \left.+\frac{1}{|\Lambda|} \sum_{j=1}^{n}\left|\mu_{j}\right| \frac{1+\left(\log \xi_{j}\right)^{\beta_{j}+q}}{\Gamma\left(\beta_{j}+q+1\right)}\right)
\end{aligned}
$$$$
=\|\kappa\| \Phi \leq \bar{r} .
$$

This shows that $\mathscr{P} x+\mathcal{Q} y \in B_{\bar{r}}$. It follows from assumption $\left(\mathrm{H}_{1}\right)$ together with $(24)$ that $Q$ is a contraction mapping. Since the function $f$ is continuous, we have that the operator $\mathscr{P}$ is continuous. It is easy to verify that

$$
\begin{aligned}
\|\mathscr{P} x\| \leq\|\kappa\| & \frac{1}{|\Lambda|} \sum_{i=1}^{m}\left|\lambda_{i}\right| \frac{\left(\log \eta_{i}\right)^{\alpha_{i}+q}}{\Gamma\left(\alpha_{i}+q+1\right)} \\
& \left.+\frac{1}{|\Lambda|} \sum_{j=1}^{n}\left|\mu_{j}\right| \frac{1+\left(\log \xi_{j}\right)^{\beta_{j}+q}}{\Gamma\left(\beta_{j}+q+1\right)}\right) .
\end{aligned}
$$

Therefore, $\mathscr{P}$ is uniformly bounded on $B_{\bar{r}}$.

Next, we prove the compactness of the operator $\mathscr{P}$. Let us set $\sup _{(t, x) \in[1, e] \times B_{\bar{r}}}|f(t, x)|=\bar{f}<\infty$; consequently we get

$$
\begin{aligned}
& \left|(\mathscr{P} x)\left(t_{1}\right)-(\mathscr{P} x)\left(t_{2}\right)\right| \\
& =\mid \frac{\left(\log t_{1}\right)^{q-1}}{\Lambda} \sum_{j=1}^{n} \mu_{j}\left(J^{\beta_{j}+q} f(s, x(s))(e)\right. \\
& -\frac{\left(\log t_{1}\right)^{q-1}}{\Lambda} \sum_{i=1}^{m} \lambda_{i} J^{\alpha_{i}+q} f(s, x(s))\left(\eta_{i}\right) \\
& -\frac{\left(\log t_{2}\right)^{q-1}}{\Lambda} \sum_{j=1}^{n} \mu_{j}\left(J^{\beta_{j}+q} f(s, x(s))(e)\right. \\
& +\frac{\left(\log t_{2}\right)^{q-1}}{\Lambda} \sum_{i=1}^{m} \lambda_{i} J^{\alpha_{i}+q} f(s, x(s))\left(\eta_{i}\right) \mid \\
& \leq \bar{f} \frac{\left(\log t_{2}\right)^{q-1}-\left(\log t_{1}\right)^{q-1} \mid m}{|\Lambda|} \sum_{i=1}^{m}\left|\lambda_{i}\right| \frac{\left(\log \eta_{i}\right)^{\alpha_{i}+q}}{\Gamma\left(\alpha_{i}+q+1\right)} \\
& +|\Lambda|
\end{aligned}
$$

which is independent of $x$ and tends to zero as $t_{2} \rightarrow t_{1}$. Thus, $\mathscr{P}$ is equicontinuous. So $\mathscr{P}$ is relatively compact on $B_{\bar{r}}$. Hence, by the Arzelá-Ascoli theorem, $\mathscr{P}$ is compact on $B_{\bar{r}}$. Thus, all the assumptions of Lemma 8 are satisfied. So the boundary value problem (1)-(2) has at least one solution on $[1, e]$. The proof is completed.

Remark 10. In the above theorem we can interchange the roles of the operators $\mathscr{P}$ and $Q$ to obtain a second result replacing (24) by the following condition:

$$
\frac{L}{|\Lambda|} \sum_{i=1}^{m}\left|\lambda_{i}\right| \frac{\left(\log \eta_{i}\right)^{\alpha_{i}+q}}{\Gamma\left(\alpha_{i}+q+1\right)}+\frac{L}{|\Lambda|} \sum_{j=1}^{n}\left|\mu_{j}\right| \frac{1+\left(\log \xi_{j}\right)^{\beta_{j}+q}}{\Gamma\left(\beta_{j}+q+1\right)}<1 .
$$

Now, our last existence result is based on Leray-Schauder's nonlinear alternative.

Theorem 11 (nonlinear alternative for single-valued maps [31]). Let $E$ be a Banach space, $C$ a closed, convex subset of $E$, $U$ an open subset of $C$, and $0 \in U$. Suppose that $F: \bar{U} \rightarrow C$ is a continuous, compact (i.e., $F(\bar{U})$ is a relatively compact subset of C) map. Then either

(i) F has a fixed point in $\bar{U}$ or

(ii) there is a $u \in \partial U$ (the boundary of $U$ in $C$ ) and $\lambda \epsilon$ $(0,1)$, with $u=\lambda F(u)$.

Theorem 12. Assume that $f:[1, e] \times \mathbb{R} \rightarrow \mathbb{R}$ is a continuous function. In addition we suppose that

$\left(\mathrm{H}_{4}\right)$ there exists a continuous nondecreasing function $\psi$ : $[0, \infty) \rightarrow(0, \infty)$ and a function $p \in C\left([1, e], \mathbb{R}^{+}\right)$ such that

$|f(t, x)| \leq p(t) \psi(|x|) \quad$ for each $(t, x) \in[1, e] \times \mathbb{R}$

$\left(\mathrm{H}_{5}\right)$ there exists a constant $N>0$ such that

$$
\frac{N}{\|p\| \psi(N) \Phi}>1
$$

where $\Phi$ is defined by (15).

Then the boundary value problem (1)-(2) has at least one solution on $[1, e]$.

Proof. Firstly, we will show that the operator $\mathscr{F}$, defined by (14), maps bounded sets (balls) into bounded sets in $\mathscr{C}$. For a 
positive number $R$, let $B_{R}=\{x \in \mathscr{C}:\|x\| \leq R\}$ be a bounded ball in $\mathscr{C}$. Then for $t \in[1, e]$, we have

$$
\begin{aligned}
|\mathscr{F} x(t)| \leq & J^{q}|f(s, x(s))|(e) \\
& +\frac{1}{|\Lambda|} \sum_{i=1}^{m}\left|\lambda_{i}\right| J^{\alpha_{i}+q}|f(s, x(s))|\left(\eta_{i}\right) \\
& +\frac{1}{|\Lambda|} \sum_{j=1}^{n}\left|\mu_{j}\right|\left(J^{\beta_{j}+q}|f(s, x(s))|(e)\right. \\
\leq & \|p\| \psi(\|x\|) \frac{1}{\Gamma(q+1)} \\
& +\|p\| \psi(\|x\|) \frac{1}{|\Lambda|} \sum_{i=1}^{m}\left|\lambda_{i}\right| \frac{\left(\log \eta_{i}\right)^{\alpha_{i}+q}}{\Gamma\left(\alpha_{i}+q+1\right)} \\
& +\|p\| \psi(\|x\|) \frac{1}{|\Lambda|} \sum_{j=1}^{n}\left|\mu_{j}\right| \frac{1+\left(\log \xi_{j}\right)^{\beta_{j}+q}}{\Gamma\left(\beta_{j}+q+1\right)} \\
\leq & \|p\| \psi(R) \frac{1}{\Gamma(q+1)} \\
& +\|p\| \psi(R) \frac{1}{|\Lambda|} \sum_{i=1}^{m}\left|\lambda_{i}\right| \frac{\left(\log \eta_{i}\right)^{\alpha_{i}+q}}{\Gamma\left(\alpha_{i}+q+1\right)} \\
& +\|p\| \psi(R) \frac{1}{|\Lambda|} \sum_{j=1}^{n}\left|\mu_{j}\right| \frac{1+\left(\log \xi_{j}\right)^{\beta_{j}+q}}{\Gamma\left(\beta_{j}+q+1\right)} \\
= & K .
\end{aligned}
$$

Therefore, we conclude that $\|\mathscr{F} x\| \leq K$.

Secondly, we show that $\mathscr{F}$ maps bounded sets into equicontinuous sets of $\mathscr{C}$. Let $\sup _{(t, x) \in[1, e] \times B_{R}}|f(t, x)|=f^{*}<\infty$, $v_{1}, v_{2} \in[1, e]$ with $v_{1}<v_{2}$ and $x \in B_{R}$. Then we have

$$
\begin{aligned}
& \left|(\mathscr{F} x)\left(v_{2}\right)-(\mathscr{F} x)\left(v_{1}\right)\right| \\
& =\mid J^{q} f(s, x(s))\left(v_{2}\right) \\
& \quad-\frac{\left(\log v_{2}\right)^{q-1}}{\Lambda} \sum_{i=1}^{m} \lambda_{i} J^{\alpha_{i}+q} f(s, x(s))\left(\eta_{i}\right) \\
& +\frac{\left(\log v_{2}\right)^{q-1}}{\Lambda} \sum_{j=1}^{n} \mu_{j}\left(J^{\beta_{j}+q} f(s, x(s))(e)\right. \\
& \quad-J^{q} f(s, x(s))\left(\nu_{1}\right) \\
& +\frac{\left(\log v_{1}\right)^{q-1}}{\Lambda} \sum_{i=1}^{m} \lambda_{i} J^{\alpha_{i}+q} f(s, x(s))\left(\eta_{i}\right)
\end{aligned}
$$

$$
\begin{gathered}
-\frac{\left(\log \nu_{1}\right)^{q-1}}{\Lambda} \sum_{j=1}^{n} \mu_{j}\left(J^{\beta_{j}+q} f(s, x(s))(e)\right. \\
\left.-J^{\beta_{j}+q} f(s, x(s))\left(\xi_{j}\right)\right) \mid \\
\leq f^{*} \frac{\left|\left(\log \nu_{2}\right)^{q}-\left(\log \nu_{1}\right)^{q}\right|}{\Gamma(q+1)} \\
+f^{*} \frac{\left|\left(\log \nu_{2}\right)^{q-1}-\left(\log \nu_{1}\right)^{q-1}\right|}{|\Lambda|} \sum_{i=1}^{m}\left|\lambda_{i}\right| \frac{\left(\log \eta_{i}\right)^{\alpha_{i}+q}}{\Gamma\left(\alpha_{i}+q+1\right)} \\
+f^{*} \frac{\left|\left(\log \nu_{2}\right)^{q-1}-\left(\log \nu_{1}\right)^{q-1}\right|}{|\Lambda|} \sum_{j=1}^{n}\left|\mu_{j}\right| \frac{1-\left(\log \xi_{j}\right)^{\beta_{j}+q}}{\Gamma\left(\beta_{j}+q+1\right)} .
\end{gathered}
$$

Obviously, the right hand side of the above inequality tends to zero independently of $x \in B_{R}$ as $\nu_{2} \rightarrow v_{1}$. Therefore it follows from the Arzelá-Ascoli theorem that $\mathscr{F}: \mathscr{C} \rightarrow \mathscr{C}$ is completely continuous.

Let $x$ be a solution. Then, for $t \in[1, e]$, following the similar computations as in the first step, we have

$$
\begin{aligned}
\|x\| \leq & \|p\| \psi(\|x\|) \frac{1}{\Gamma(q+1)} \\
& +\|p\| \psi(\|x\|) \frac{1}{|\Lambda|} \sum_{i=1}^{m}\left|\lambda_{i}\right| \frac{\left(\log \eta_{i}\right)^{\alpha_{i}+q}}{\Gamma\left(\alpha_{i}+q+1\right)} \\
& +\|p\| \psi(\|x\|) \frac{1}{|\Lambda|} \sum_{j=1}^{n}\left|\mu_{j}\right| \frac{1+\left(\log \xi_{j}\right)^{\beta_{j}+q}}{\Gamma\left(\beta_{j}+q+1\right)} \\
= & \|p\| \psi(\|x\|) \Phi .
\end{aligned}
$$

Consequently, we have

$$
\frac{\|x\|}{\|p\| \psi(\|x\|) \Phi} \leq 1
$$

In view of $\left(\mathrm{H}_{5}\right)$, there exists $N$ such that $\|x\| \neq N$. Let us set

$$
U=\{x \in \mathscr{C}:\|x\|<N\}
$$

Note that the operator $\mathscr{F}: \bar{U} \rightarrow \mathscr{C}$ is continuous and completely continuous. From the choice of $U$, there is no $x \in \partial U$ such that $x=\theta \mathscr{F} x$ for some $\theta \in(0,1)$. Consequently, by nonlinear alternative of Leray-Schauder type (Theorem 11) we deduce that $\mathscr{F}$ has a fixed point in $\bar{U}$, which is a solution of the boundary value problem (1)-(2). This completes the proof. 


\section{Examples}

Example 1. Consider the following boundary value problem for Hadamard fractional differential equation:

$$
\begin{aligned}
& D^{3 / 2} x(t)=\frac{\log t^{5}}{e^{t}(t+2)^{2}} \frac{|x(t)|}{(3+|x(t)|)}, \quad t \in J=[1, e] \\
& x(1)=0 \\
& 2 J^{1 / 4} x\left(\frac{5}{4}\right)+\frac{1}{5} J^{3 / 2} x\left(\frac{9}{5}\right)+3 J^{2} x\left(\frac{15}{7}\right) \\
& =J^{2 / 3} x(e)-J^{2 / 3} x\left(\frac{10}{7}\right)+5\left(J^{9 / 7} x(e)-J^{9 / 7} x(2)\right) \\
& -2\left(J^{11 / 4} x(e)-J^{11 / 4} x\left(\frac{9}{4}\right)\right) .
\end{aligned}
$$

Here $q=3 / 2, \lambda_{1}=2, \lambda_{2}=1 / 5, \lambda_{3}=3, \alpha_{1}=1 / 4, \alpha_{2}=$ $3 / 2, \alpha_{3}=2, \eta_{1}=5 / 4, \eta_{2}=9 / 5, \eta_{3}=15 / 7, \mu_{1}=1, \mu_{2}=$ $5, \mu_{3}=-2, \beta_{1}=2 / 3, \beta_{2}=9 / 7, \beta_{3}=11 / 4, \xi_{1}=10 / 7, \xi_{2}=$ $2, \xi_{3}=9 / 4$, and $f(t, x)=\left(\log t^{5}|x|\right) /\left(e^{t}(t+2)^{2}(3+|x|)\right)$. Since

$$
|f(t, x)-f(t, y)| \leq\left(\frac{5}{27 e}\right)|x-y|
$$

then $\left(\mathrm{H}_{1}\right)$ is satisfied with $L=5 / 27 e$. We can show that

$$
\begin{aligned}
\Lambda= & \sum_{i=1}^{m} \lambda_{i} \frac{\Gamma(q)}{\Gamma\left(q+\alpha_{i}\right)}\left(\log \eta_{i}\right)^{q+\alpha_{i}-1} \\
& -\sum_{j=1}^{n} \mu_{j} \frac{\Gamma(q)}{\Gamma\left(q+\beta_{j}\right)}\left(1-\left(\log \xi_{j}\right)^{q+\beta_{j}-1}\right) \\
\approx & -0.6895040549, \\
\Phi= & \frac{1}{\Gamma(q+1)}+\frac{1}{|\Lambda|} \sum_{i=1}^{m}\left|\lambda_{i}\right| \frac{\left(\log \eta_{i}\right)^{\alpha_{i}+q}}{\Gamma\left(\alpha_{i}+q+1\right)} \\
& +\frac{1}{|\Lambda|} \sum_{j=1}^{n}\left|\mu_{j}\right| \frac{1+\left(\log \xi_{j}\right)^{\beta_{j}+q}}{\Gamma\left(\beta_{j}+q+1\right)} \\
\approx & 3.975680952, \\
L \Phi= & \frac{5}{27 e}(3.975680952) \approx 0.2708465347<1 .
\end{aligned}
$$

Hence, by Theorem 4, the boundary value problem (38) has a unique solution on $[1, e]$.
Example 2. Consider the following boundary value problem for Hadamard fractional differential equation:

$$
\begin{aligned}
& D^{7 / 4} x(t)= \frac{e^{t}}{(t+1)^{2}} \frac{|x(t)|}{(2+|x(t)|)}, \quad t \in J=[1, e], \\
& x(1)=0 \\
& \frac{1}{4} J^{6 / 7} x\left(\frac{7}{3}\right)-\frac{2}{3} J^{3} x\left(\frac{7}{5}\right)-2 J^{5 / 2} x(2) \\
&=4\left(J^{5} x(e)-J^{5} x\left(\frac{11}{5}\right)\right) \\
&+\frac{11}{4}\left(J^{3 / 4} x(e)-J^{3 / 4} x\left(\frac{16}{13}\right)\right) .
\end{aligned}
$$

Here $q=7 / 4, \lambda_{1}=1 / 4, \lambda_{2}=-2 / 3, \lambda_{3}=-2, \alpha_{1}=6 / 7$, $\alpha_{2}=3, \alpha_{3}=5 / 2, \eta_{1}=7 / 3, \eta_{2}=7 / 5, \eta_{3}=2, \mu_{1}=4, \mu_{2}=$ $11 / 4, \beta_{1}=5, \beta_{2}=3 / 4, \xi_{1}=11 / 5, \xi_{2}=16 / 13$, and $f(t, x)=$ $\left(e^{t}|x|\right) /\left((t+1)^{2}(2+|x|)\right)$. We choose $h(t)=e^{t} / 4$ and that

$$
\begin{aligned}
\Lambda= & \sum_{i=1}^{m} \lambda_{i} \frac{\Gamma(q)}{\Gamma\left(q+\alpha_{i}\right)}\left(\log \eta_{i}\right)^{q+\alpha_{i}-1} \\
& -\sum_{j=1}^{n} \mu_{j} \frac{\Gamma(q)}{\Gamma\left(q+\beta_{j}\right)}\left(1-\left(\log \xi_{j}\right)^{q+\beta_{j}-1}\right) \\
\approx & -1.672972140, \\
H^{*}= & J^{q} h(e)+\frac{1}{|\Lambda|} \sum_{i=1}^{m}\left|\lambda_{i}\right| J^{\alpha_{i}+q} h\left(\eta_{i}\right) \\
& +\frac{1}{|\Lambda|} \sum_{j=1}^{n}\left|\mu_{j}\right|\left(J^{\beta_{j}+q} h(e)+J^{\beta_{j}+q} h\left(\xi_{j}\right)\right) \\
\approx & 1.295076743 .
\end{aligned}
$$

Clearly,

$$
\begin{aligned}
|f(t, x)-f(t, y)| & =\frac{e^{t}}{(1+t)^{2}}\left(\frac{2|x|-2|y|}{4+2|x|+2|y|+|x||y|}\right) \\
& \leq \frac{e^{t}}{4}\left(\frac{|x-y|}{1.295076743+|x-y|}\right) .
\end{aligned}
$$

Hence, by Theorem 7, the boundary value problem (41) has a unique solution on $[1, e]$. 
Example 3. Consider the following boundary value problem for Hadamard fractional differential equation:

$$
\begin{aligned}
& D^{6 / 5} x(t)=\frac{2 \sin (x / 4)}{5 \pi+\left(e^{x}+1\right)^{2}}+\frac{2+\cos (\pi t)}{10 \pi+3}, \quad t \in J=[1, e], \\
& x(1)=0, \\
& J^{4} x\left(\frac{3}{2}\right)-3 J^{9 / 4} x(2)-10 J^{1 / 5} x\left(\frac{7}{4}\right)+6 J^{7 / 2} x\left(\frac{5}{2}\right) \\
& +\frac{14}{3} J^{5} x\left(\frac{11}{9}\right) \\
& =3\left(J^{3 / 2} x(e)-J^{3 / 2} x\left(\frac{11}{7}\right)\right)-7\left(J^{3} x(e)-J^{3} x\left(\frac{17}{13}\right)\right) \\
& +\frac{4}{3}\left(J^{5 / 3} x(e)-J^{5 / 3} x(2)\right) .
\end{aligned}
$$

Here $q=6 / 5, \lambda_{1}=1, \lambda_{2}=-3, \lambda_{3}=-10, \lambda_{4}=6, \lambda_{5}=$ $14 / 3, \alpha_{1}=4, \alpha_{2}=9 / 4, \alpha_{3}=1 / 5, \alpha_{4}=7 / 2, \alpha_{5}=5, \eta_{1}=$ $3 / 2, \eta_{2}=2, \eta_{3}=7 / 4, \eta_{4}=5 / 2, \eta_{5}=11 / 9, \mu_{1}=3, \mu_{2}=$ $-7, \mu_{3}=4 / 3, \beta_{1}=3 / 2, \beta_{2}=3, \beta_{3}=5 / 3, \xi_{1}=11 / 7, \xi_{2}=$ $17 / 13, \xi_{3}=2$, and $f(t, x)=(2 \sin (x / 4)) /\left(5 \pi+\left(e^{x}+1\right)^{2}\right)+$ $(2+\cos (\pi t)) /(10 \pi+3)$. Clearly,

$$
\begin{aligned}
|f(t, x)| & =\left|\frac{2 \sin (x / 4)}{5 \pi+\left(e^{x}+1\right)^{2}}+\frac{2+\cos (\pi t)}{10 \pi+3}\right| \\
& \leq(2+\cos (\pi t))\left(\frac{|x|+1}{10 \pi}\right) .
\end{aligned}
$$

Choosing $p(t)=2+\cos (\pi t)$ and $\psi(|x|)=(|x|+1) /(10 \pi)$, we can show that

$$
\begin{aligned}
\Lambda= & \sum_{i=1}^{m} \lambda_{i} \frac{\Gamma(q)}{\Gamma\left(q+\alpha_{i}\right)}\left(\log \eta_{i}\right)^{q+\alpha_{i}-1} \\
& -\sum_{j=1}^{n} \mu_{j} \frac{\Gamma(q)}{\Gamma\left(q+\beta_{j}\right)}\left(1-\left(\log \xi_{j}\right)^{q+\beta_{j}-1}\right) \\
\approx & -9.148087406, \\
\Phi= & \frac{1}{\Gamma(q+1)}+\frac{1}{|\Lambda|} \sum_{i=1}^{m}\left|\lambda_{i}\right| \frac{\left(\log \eta_{i}\right)^{\alpha_{i}+q}}{\Gamma\left(\alpha_{i}+q+1\right)} \\
& +\frac{1}{|\Lambda|} \sum_{j=1}^{n}\left|\mu_{j}\right| \frac{1+\left(\log \xi_{j}\right)^{\beta_{j}+q}}{\Gamma\left(\beta_{j}+q+1\right)} \\
\approx & 1.462649525, \\
& \frac{N}{(3)((N+1) / 10 \pi)(1.462649525)}>1,
\end{aligned}
$$

which implies that $N>0.1623483851$. Hence, by Theorem 12 , the boundary value problem (44) has at least one solution on $[1, e]$.

\section{Conflict of Interests}

The authors declare that there is no conflict of interests regarding the publication of this paper.

\section{Acknowledgments}

The research of Phollakrit Thiramanus is supported by the Centre of Excellence in Mathematics, the Commission on Higher Education, Thailand. The research of Jessada Tariboon is supported by King Mongkut's University of Technology North Bangkok, Thailand. Sotiris K. Ntouyas is a member of Nonlinear Analysis and Applied Mathematics (NAAM) Research Group at King Abdulaziz University, Jeddah, Saudi Arabia.

\section{References}

[1] B. Ahmad, S. K. Ntouyas, and A. Alsaedi, "A study of nonlinear fractional differential equations of arbitrary order with Riemann-Liouville type multistrip boundary conditions," Mathematical Problems in Engineering, vol. 2013, Article ID 320415, 9 pages, 2013.

[2] A. A. Kilbas, H. M. Srivastava, and J. J. Trujillo, Theory and Applications of Fractional Differential Equations, vol. 204 of North-Holland Mathematics Studies, Elsevier Science B.V., Amsterdam, The Netherlands, 2006.

[3] V. Lakshmikantham, S. Leela, and J. Vasundhara Devi, Theory of Fractional Dynamic Systems, Cambridge Academic Publishers, Cambridge, UK, 2009.

[4] I. Podlubny, Fractional Differential Equations, Academic Press, San Diego, Calif, USA, 1999.

[5] S. G. Samko, A. A. Kilbas, and O. I. Marichev, Fractional Integrals and Derivatives, Theory and Applications, Gordon and Breach, Yverdon, Switzerland, 1993.

[6] M. Benchohra, S. Hamani, and S. K. Ntouyas, "Boundary value problems for differential equations with fractional order and nonlocal conditions," Nonlinear Analysis: Theory, Methods \& Applications, vol. 71, no. 7-8, pp. 2391-2396, 2009.

[7] B. Ahmad and S. K. Ntouyas, "Some existence results for boundary value problems of fractional differential inclusions with non-separated boundary conditions," Electronic Journal of Qualitative Theory of Differential Equations, vol. 71, pp. 1-17, 2010.

[8] Z. Bai, "On positive solutions of a nonlocal fractional boundary value problem," Nonlinear Analysis: Theory, Methods \& Applications, vol. 72, no. 2, pp. 916-924, 2010.

[9] K. Balachandran and J. J. Trujillo, "The nonlocal Cauchy problem for nonlinear fractional integrodifferential equations in Banach spaces," Nonlinear Analysis: Theory, Methods \& Applications, vol. 72, no. 12, pp. 4587-4593, 2010.

[10] D. Băleanu and O. G. Mustafa, "On the global existence of solutions to a class of fractional differential equations," Computers \& Mathematics with Applications, vol. 59, no. 5, pp. 1835-1841, 2010.

[11] N. J. Ford and M. L. Morgado, "Fractional boundary value problems: analysis and numerical methods," Fractional Calculus and Applied Analysis, vol. 14, no. 4, pp. 554-567, 2011. 
[12] B. Ahmad, S. K. Ntouyas, and A. Alsaedi, "New existence results for nonlinear fractional differential equations with threepoint integral boundary conditions," Advances in Difference Equations, vol. 2011, Article ID 107384, 11 pages, 2011.

[13] D. O’Regan and S. Staněk, "Fractional boundary value problems with singularities in space variables," Nonlinear Dynamics, vol. 71, no. 4, pp. 641-652, 2013.

[14] A. Debbouche, D. Baleanu, and R. P. Agarwal, "Nonlocal nonlinear integrodifferential equations of fractional orders," Boundary Value Problems, vol. 2012, article 78, 2012.

[15] Y. Chen, X. Tang, and X. He, "Positive solutions of fractional differential inclusions at resonance," Mediterranean Journal of Mathematics, vol. 10, no. 3, pp. 1207-1220, 2013.

[16] D. Băleanu, O. G. Mustafa, and R. P. Agarwal, "On the solution set for a class of sequential fractional differential equations," Journal of Physics A: Mathematical and Theoretical, vol. 43, no. 38, Article ID 385209, 2010.

[17] S. D. Purohit and S. L. Kalla, "On fractional partial differential equations related to quantum mechanics," Journal of Physics A: Mathematical and Theoretical, vol. 44, no. 4, Article ID 045202, 2011.

[18] D. Baleanu, K. Diethelm, E. Scalas, and J. J. Trujillo, Fractional Calculus Models and Numerical Methods, vol. 3 of Complexity, Nonlinearity and Chaos, World Scientific, 2012.

[19] S. D. Purohit, "Solutions of fractional partial differential equations of quantum mechanics," Advances in Applied Mathematics and Mechanics, vol. 5, no. 5, pp. 639-651, 2013.

[20] D. Baleanu, R. P. Agarwal, H. Mohammadi, and S. Rezapour, "Some existence results for a nonlinear fractional differential equation on partially ordered Banach spaces," Boundary Value Problems, vol. 2013, article 112, 8 pages, 2013.

[21] J. Hadamard, "Essai sur l'étude des fonctions données par leur développement de Taylor," Journal de Mathématiques Pures et Appliquées 4e Série, vol. 8, pp. 101-186, 1892.

[22] P. L. Butzer, A. A. Kilbas, and J. J. Trujillo, "Compositions of Hadamard-type fractional integration operators and the semigroup property," Journal of Mathematical Analysis and Applications, vol. 269, no. 2, pp. 387-400, 2002.

[23] P. L. Butzer, A. A. Kilbas, and J. J. Trujillo, "Fractional calculus in the Mellin setting and Hadamard-type fractional integrals," Journal of Mathematical Analysis and Applications, vol. 269, no. 1, pp. 1-27, 2002.

[24] P. L. Butzer, A. A. Kilbas, and J. J. Trujillo, "Mellin transform analysis and integration by parts for Hadamard-type fractional integrals," Journal of Mathematical Analysis and Applications, vol. 270, no. 1, pp. 1-15, 2002.

[25] A. A. Kilbas, "Hadamard-type fractional calculus," Journal of the Korean Mathematical Society, vol. 38, no. 6, pp. 1191-1204, 2001.

[26] A. A. Kilbas and J. J. Trujillo, "Hadamard-type integrals as Gtransforms," Integral Transforms and Special Functions, vol. 14, no. 5, pp. 413-427, 2003.

[27] B. Ahmad and S. K. Ntouyas, "On Hadamard fractional integrodifferential boundary value problems," Journal of Applied Mathematics and Computing, 2014.

[28] B. Ahmad and S. K. Ntouyas, "A fully Hadamard type integral boundary value problem of a coupled system of fractional differential equations," Fractional Calculus and Applied Analysis, vol. 17, no. 2, pp. 348-360, 2014.

[29] D. W. Boyd and J. S. W. Wong, "On nonlinear contractions," Proceedings of the American Mathematical Society, vol. 20, pp. 458-464, 1969.
[30] M. A. Krasnosel'skii, “Two remarks on the method of successive approximations," Uspekhi Matematicheskikh Nauk, vol. 10, no. 1, pp. 123-127, 1955.

[31] A. Granas and J. Dugundji, Fixed Point Theory, Springer, New York, NY, USA, 2003. 


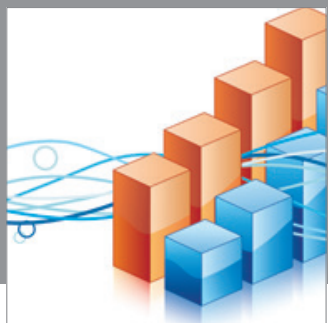

Advances in

Operations Research

mansans

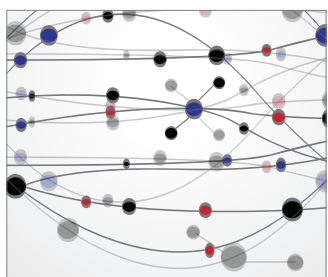

The Scientific World Journal
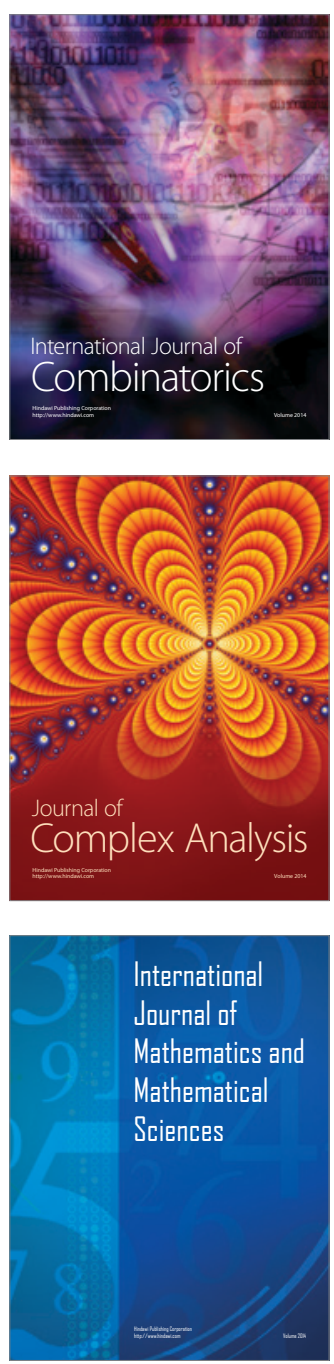
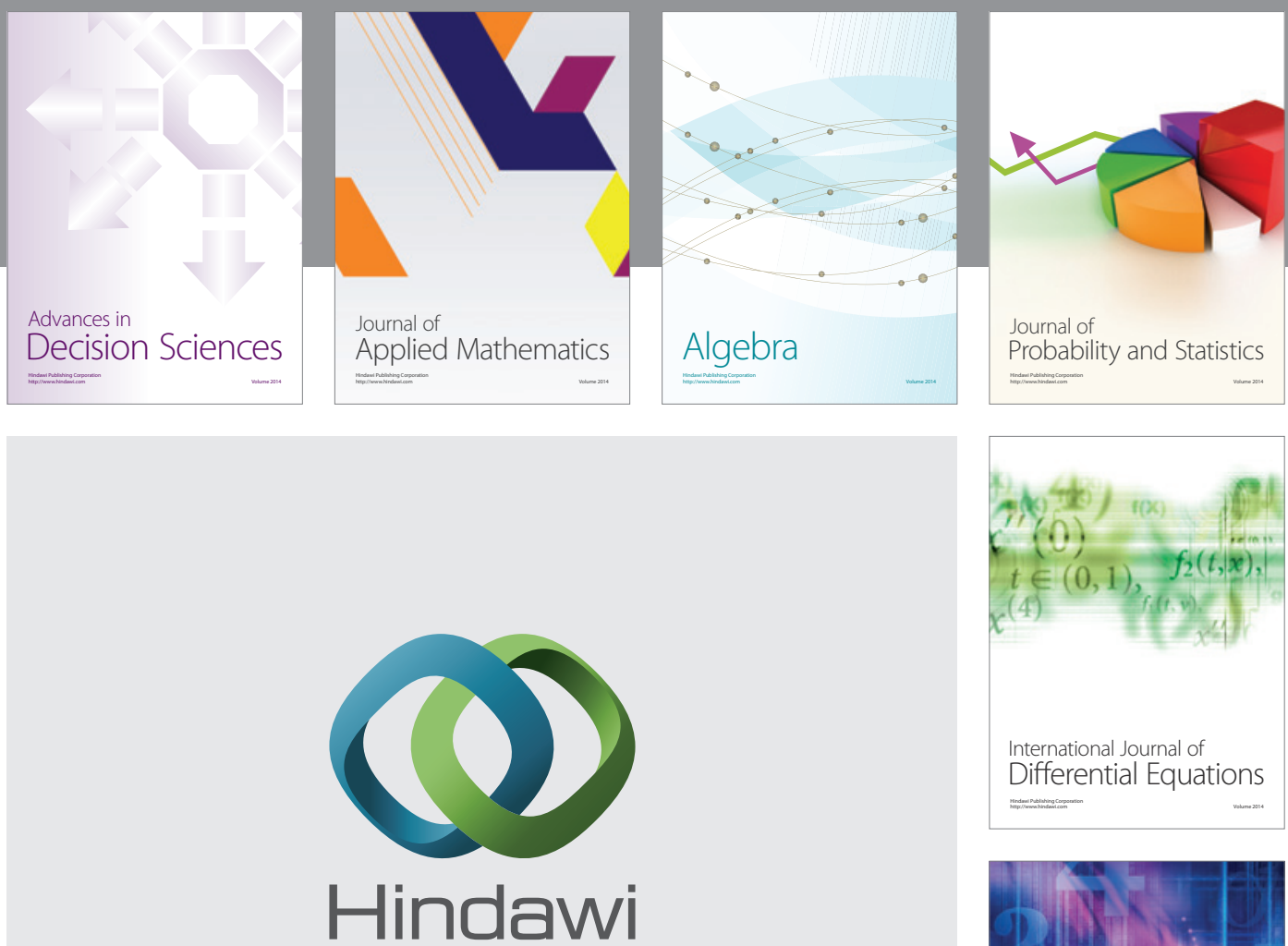

Submit your manuscripts at http://www.hindawi.com
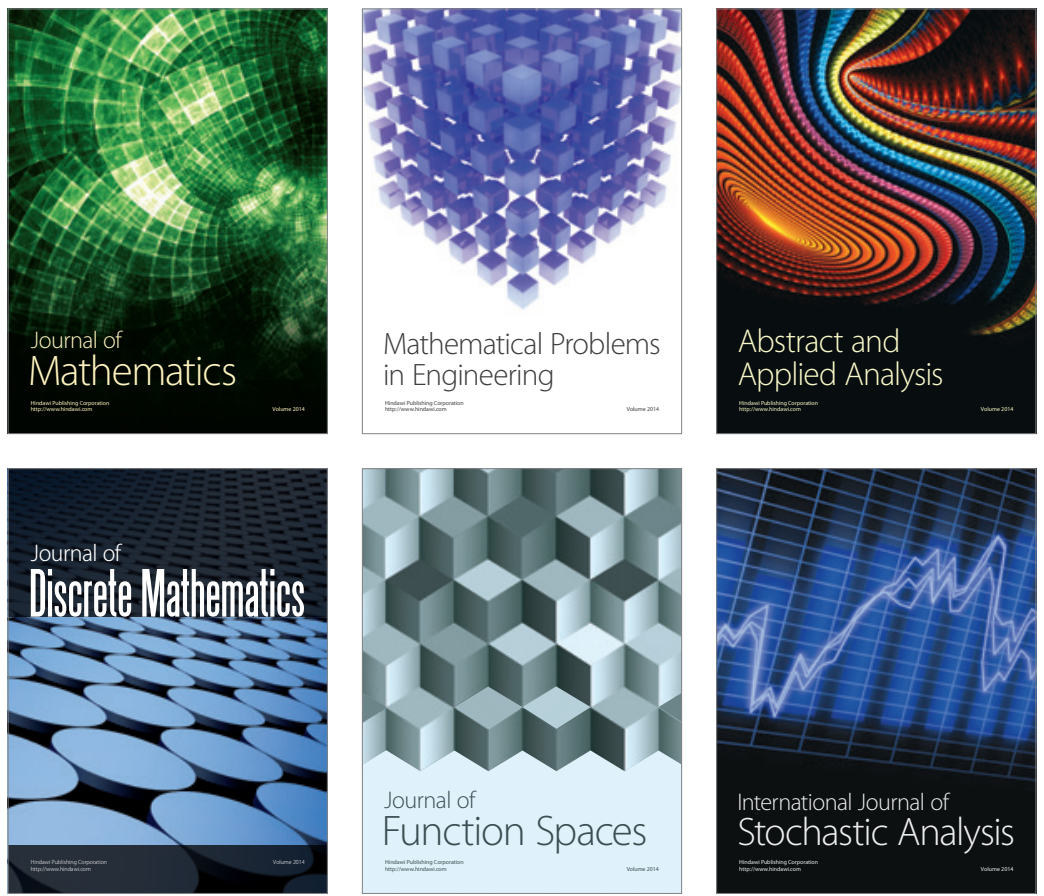

Journal of

Function Spaces

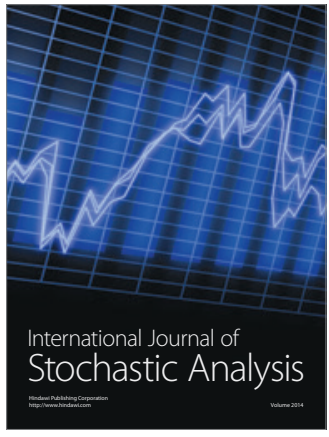

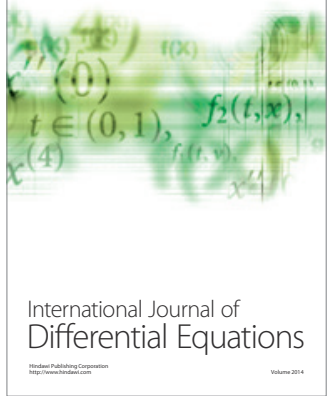
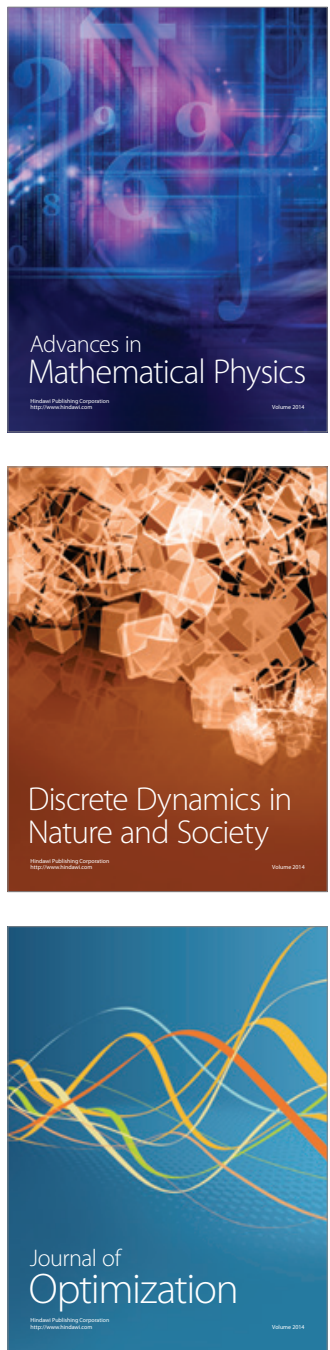